LAWRENCE

LI VERMORE NATI ONAL LABORATORY

\title{
Estimating Absolute Site Effects
}

Luca Malagnini ${ }^{1}$,Kevin Mayeda ${ }^{2}$, Aybige Akinci ${ }^{1}$ and Pier Luigi Bragato ${ }^{3}$

${ }^{1}$ Istituto Nazionale di Geofisica e Vulcanolgia, Rome, Italy

${ }^{2}$ Lawrence Livermore National Laboratory

${ }^{3}$ Istituto Nazionale di Oceanografia e di Geofisica Sperimentale, Italy

\section{January 13, 2004}

Submitted to:

Bulletin of the Seismological Society of America 


\section{DISCLAIMER}

This document was prepared as an account of work sponsored by an agency of the United States Government. Neither the United States Government nor the University of California nor any of their employees, makes any warranty, express or implied, or assumes any legal liability or responsibility for the accuracy, completeness, or usefulness of any information, apparatus, product, or process disclosed, or represents that its use would not infringe privately owned rights. Reference herein to any specific commercial product, process, or service by trade name, trademark, manufacturer, or otherwise, does not necessarily constitute or imply its endorsement, recommendation, or favoring by the United States Government or the University of California. The views and opinions of authors expressed herein do not necessarily state or reflect those of the United States Government or the University of California, and shall not be used for advertising or product endorsement purposes.

This work was performed under the auspices of the U.S. Department of Energy by the University of California, Lawrence Livermore National Laboratory under Contract No. W-7405-Eng-48. 


\title{
Estimating Absolute Site Effects
}

\author{
Luca Malagnini ${ }^{\circ}$, Kevin Mayeda ${ }^{\circ}$, Aybige Akinci $^{\circ}$, \\ and Pier Luigi Bragato ${ }^{\circ 00}$ \\ ${ }^{\circ}$ Istituto Nazionale di Geofisica e Vulcanologia, Rome, Italy \\ ${ }^{\circ}$ Lawrence Livermore National Laboratory, CA, USA \\ ${ }^{\circ}$ Istituto Nazionale di Oceanografia e di Geofisica Sperimentale, Italy
}

Submitted to Bull. Seism. Soc. Am.

January 13, 2004 


\section{Abstract}

We use previously determined direct-wave attenuation functions as well as stable, codaderived source excitation spectra to isolate the absolute S-wave site effect for the horizontal and vertical components of weak ground motion. We used selected stations in the seismic network of the eastern Alps, and find the following: (1) all "hard rock" sites exhibited deamplification phenomena due to absorption at frequencies ranging between 0.5 and $12 \mathrm{~Hz}$ (the available bandwidth), on both the horizontal and vertical components; (2) "hard rock" site transfer functions showed large variability at high-frequency; (3) vertical-motion site transfer functions show strong frequency-dependence, and (4) H/V spectral ratios do not reproduce the charactersitics of the true horizontal site transfer functions; (5) traditional, relative site terms obtained by using reference "rock sites" can be misleading in inferring the behaviors of true site transfer functions, since most rock sites have non-flat responses due to shallow heterogeneities resulting from varying degrees of weathering.

We also use our stable source spectra to estimate total radiated seismic energy and compare against previous results. We find that the earthquakes in this region exhibit nonconstant dynamic stress drop scaling which gives further support for a fundamental difference in rupture dynamics between small and large earthquakes.

To correct the vertical and horizontal S-wave spectra for attenuation, we used detailed regional attenuation functions derived by Malagnini et al. (2002) who determined frequencydependent geometrical spreading and $\mathrm{Q}$ for the region. These corrections account for the gross path effects (i.e., all distance-dependent effects), although the source and site effects are still present in the distance-corrected spectra. The main goal of this study is to isolate the absolute site effect (as a function of frequency) by removing the source spectrum (momentrate spectrum) from the distance-corrected S-wave spectra. Typically, removing the S-wave source spectrum is difficult because of inadequate corrections for the source radiation pattern, directivity and random interference. In addition to complexities near the source, 2-D and 3-D structure beneath the recording site will result in an azimuth-dependent site effect. Since the direct wave only samples a narrow range in take-off and back-azimuth angles, multi-station 
averaging is needed to minimize the inherent scatter.

To minimize these complicating effects, we apply the coda methodology outlined by Mayeda et al., (2003) to obtain stable moment-rate spectra. This methodology provides source amplitude and derived source spectra that are a factor of 3-to-4 times more stable than those derived from direct waves. Since the coda is commonly thought of as scattered energy that samples all ray parameters and back-azimuths, it is not very sensitive to the source radiation pattern and $3-\mathrm{D}$ structure. This property makes it an excellent choice for use in obtaining average properties of the source, site and path effects in a region. Due to the characteristics of the techniques used in this study, all the inverted quantities are azimuthally averaged, since the azimuthal information is lost in the processing.

\section{Introduction}

With the advent of modern instruments, accurate estimates of radiated energy from seismic sources are now possible (Mayeda and Walter, 1996, Mayeda et al., 2003). These and related measurements of scalar moment are very important in seismology, since their analysis can shed light over some basic properties of the static and dynamic behaviors of rupturing faults. These quantities represent the mere dimensions of the fault times the fault displacement (the seismic moment), and the amount of stress dynamically released while rupturing (the radiated energy).

On the one hand, a number of authors believe that larger earthquakes are characterized by an increased percentage of their total energy available for radiation with respect to smaller events, a phenomenon due to various possible physical mechanisms such as elastodynamic lubrication, pressurization of fluids, melt, etc. (see for example Kanamori and Heaton, 2000; Brodsky and Kanamori, 2001). On the other hand, other authors (e.g., Ide and Beroza, 2001) believe that the percentage of energy available for radiation is fixed and does not vary significantly with magnitude. Underlying this academic battle, fought over measurements taken even over the same data sets, are difficulties in measuring the total radiated energy from spatially sparse observations that may be strongly contaminated by the filtering actions

of the various portions of the Earth through which the seismic waves travel. Moreover, 
the strongest effects are those induced by the shallow geology. Thus, whenever recordings of ground motions are analyzed at high-frequency (i.e., $f \geq 0.5 \mathrm{~Hz}$ ), results are strongly biased by site conditions. Unfortunately, since the shallow geology is in general extremely heterogeneous in three dimensions, all attempts to completely deconvolve the site from the seismograms are likely to be grossly oversimplified.

An interesting debate exists on quantifying the typical depth controlling the seismic behaviors of sites. Usually, in engineering site investigations, $30 \mathrm{~m}$ is considered to be a good measure of this characteristic quantity. For example, Boore et al. $(1993,1994)$ based their regressions for ground motion on the average shear-wave velocity in the first $30 \mathrm{~m}$ from the free surface, and there are studies (see, for example, Borcherdt, 1992, 1994, or Martin and Dobry, 1994) that indicate the need that design of structures be based on properties of geology in the first $30 \mathrm{~m}$. A similar perspective is that by Anderson et al. (1996): after analyzing results of theoretical modeling of wave propagation in layered media, they stated the importance of resonances in shallow geologic formations, but concluded that the influence of the attenuation of the surface layer (30 m-thick) on ground motions is on the same order as that of the attenuation in the underlying layers, down to the 5-km-deep halfspace. Thus site response may be affected by a significant portion of the crust near the site.

Perfect reference sites (locations characterized by unit responses, flat throughout the entire frequency band of interest) would require uniform, perfectly elastic halfspaces. Conversely, if we had estimates of absolute site terms, we might be able to roughly quantify the depths of interest affecting a range of frequencies.

The availability of absolute source spectra and of carefully calibrated crustal propagation functions allows a new approach to the problem of estimating "absolute" site effects. By "absolute", we mean the response of the shallow geology beneath the recording sites, averaged over the suite of available azimuths and incidence angles, in terms of two competing effects: the amplifying action induced by impedance reductions at shallow depths and the severe anelastic damping occurring right below the free surface. The latter effect was characterized by Anderson and Hough (1984) with the parameter $\kappa$. 
Crustal functional forms closely describing the average attenuation of seismic waves as a function of frequency and hypocentral distance have been empirically obtained in different regions of the world (e.g., Malagnini et al., 2000, 2002, Akinci et al., 2001, Raoof et al., 1999). By means of a technique similar to that described by Malagnini et al. (2000, 2002), absolute source spectra and crustal propagation functions can be used to produce "absolute" site terms. Although the importance of the azimuthal variability of site responses has been recognized in the literature (e.g., Papageorgiou and Aki, 1983), the technique described in this study is able to quantify only the average behavior, over all the available angles of incidence and azimuths. Systematic variability of site response over azimuths and incidence angles must be investigated in detail with dedicated studies using different techniques.

In this study we address a number of open questions about absolute site terms:

- i) do "ideal" reference sites actually exist?

- ii) can we easily estimate site transfer functions by merely computing spectral ratios with respect to a reference site?

- iii) do horizontal-to-vertical ratios actually tell us anything about absolute site behavior?

Starting with the pioneering works by King and Tucker (1984) and Tucker and King (1984), many studies addressed the issues listed above, starting from the assumption that rock outcrops would be unaffected by "strange" behavior, their response being flat and of unit amplitude, although other authors (e.g., Steidl et al., 1996) already pointed out that rock sites may seriously distort the spectra of incoming seismic waves, resulting in biased estimates of site effects.

The proposition: "A rock site equals a good reference site" is certainly true at very low frequency (i.e., at periods of tens of seconds) where wavelengths are extremely long, and thus are unaffected by the shallow heterogeneities. More precisely, any site is a good reference at low enough frequencies, but for rock sites the "low frequency" cut-off is higher 
than the corresponding limit for soil sites. At intermediate and high frequencies $(f \geq 1.0$ $\mathrm{Hz}$ ) the effects of three-dimensional heterogeneities may become important even at rock sites, with the competition between higher absorption (attenuation, deamplification) and impedance contrasts/gradients (reverberations, amplifications) due to weathering, possibly creating complex behaviors, basically unpredictable, whose interpretation can be achieved only after gathering detailed information about the 3-D velocity-attenuation structure beneath each site.

\section{Data}

We used 206 events recorded by the Friuli-Venezia Giulia Seismic Network (FVGSN) (a regional network run by the Centro di Ricerche Sismologiche, CRS, of the Istituto Nazionale di Oceanografia e di Geofisica Sperimentale) selected from the larger set of about 1800 events used by Malagnini et al. (2002). All sites shown in this study, except for UDI, share the same setup: a hole is dug into the ground to reach the underlying rock formation $(\max 1.5$ $m$ deep at DRE), a concrete platform is built at the bottom of the hole, and concrete walls are used to finish it (steel housing is used at ZOU).

UDI is in a 1 m-deep hole in the backyard of CRS in Udine, which does not reach the underlying flysch rocks, that are well below $100 \mathrm{~m}$ in depth: although there is no geotechnical information at the instrumented site, a borehole on a similar site was drilled about $1 \mathrm{~km}$ away, where coarse gravels about $15 \mathrm{~m}$ thick overlay $90 \mathrm{~m}$ of gravel conglomerate which, in turn, is above clays. This 122 m-deep borehole did not reach the flysch bedrock.

ZOU (Zoufplan) is on a flat and very rigid rock outcrop (dolomite). Conditions at stations DRE (marly sandstone outcrop), CAE and BAD (limestone outcrops) do not need further specific descriptions. For details of the original data set we refer the reader to Malagnini et al. (2002). Figure 1 shows the region covered by the FVGSN, with the stations and the earthquakes used for this study. Stations CAE, ZOU, BAD and DRE are equipped with three-component seismometers. Recordings from the other stations are only from vertical-

component instruments. A gross description of the geology of sites and of the seismic stations is also given in Table 1 of Malagnini et al. (2002). 
For each of the 206 events shown in the map, we obtained an unbiased moment-rate spectrum, as described in the following paragraphs. Earthquakes were chosen based on their signal-to-noise ratios, although some small events were also included to be used as empirical Green's functions for the coda calibration.

\section{Moment-Rate Spectra - Radiated Energies - Dynamic Stress Drops}

Mayeda and Walter (1996) used the well-established insensitivity of seismic coda to source radiation pattern and to heterogeneous path effects, and produced stable moment-rate spectra for regional earthquakes, even from single-station observations. The technique was based upon a 2-D multiple-scattering model (Shang and Gao, 1988) but, due to its simplicity, additional ad-hoc path corrections were added. More recently, Mayeda et al. (2003) modified their approach to make it completely empirical, independent of any scattering model.

A comparison between direct-wave and coda-wave interstation amplitudes, is shown in Figure 2 (3.0-4.0 Hz and 8.0-10.0 ranges). Distance corrections for direct waves are obtained by using the attenuation model proposed by Malagnini et al. (2002). Interstation standard deviations for coda amplitudes are a factor of three smaller than those computed on directwaves. Plots of coda-derived amplitudes in Figure 2 clearly show the relative site term between station ZOU and station DRE at the two central frequencies.

Figure 3 shows examples of dimensionless spectra, made of distance-corrected coda amplitude measurements. Dimensionless spectra are station-dependent, (Figure 3 refers to station BAD), since they are contaminated by the station-specific site effect and by the S-to-coda transfer function. Independent estimates of spectral amplitudes of a number of events are essential for determining the combined effect of site response and S-to-coda transfer function at each station (to be applied to each distance-corrected measurement). In order to do so, we were able to find three events, recorded also at the very broadband station TRI (Trieste, part of the MedNet network, see Figure 1) which we could waveform-model. In order to do so, we used the code wvfmtd96 (Randall et al., 1995) which is now included in the last version of the package "Computer Programs for Seismology" (Herrmann, 2002). The events (listed in order of decreasing size) occurred on April 12, 1998, May 6, 1996, and May 28, 
1998. Figure 3 shows the waveform fits for the three events.

After determining the station corrections to the coda amplitude measurements due to site effects, as well as those related to S-to-coda transfer functions, the small events shown in Figure 3 were used as empirical Green's functions (EGF) in order to obtain high-frequency $(f>2.0 \mathrm{~Hz})$ spectral corrections. After all corrections are applied, we obtained "absolute" moment-rate spectra for the 206 events.

Using the Malagnini et al. (2002) crustal attenuation for Northeastern Italy, we wrote an automatic procedure to estimate moment magnitudes for the earthquakes recorded by the FVGSN. Such an automatic tool is based on the ability of deconvolving the regional average of the geometric and anelastic attenuation from the spectra of S-wave horizontal ground displacement.

Since the coda $M_{w}$ 's are tied to independent estimates of seismic moment, they represent a reference. In Figure 4 we compare the direct-wave estimates of moment magnitude against them. Although Figure 4 shows a satisfactory agreement between the coda and the direct-wave moment magnitudes, there is a small systematic dc-shift for magnitudes smaller than $M_{w}=3.2$ (direct-wave estimates are larger than coda-related ones). One possible explanation is that the direct-wave approach is affected by a subjective choice of the filters for the removal of microseisms from the recordings of the small earthquakes.

For a number of events, the corner frequency is clearly recognizable, and the estimate of radiated energy for such earthquakes can be obtained after extrapolating the corresponding coda-based spectra to DC and to infinite frequencies using an $\omega^{2}$ spectral model. When a spectrum of this data set comprises at least $70 \%$ of the total radiated energy, this quantity is plotted in deep blue in Figure 4, vs. the corresponding seismic moment. Light blue symbols indicate estimates of energy between $50 \%$ and $70 \%$ of the total, for events of this data set. Red symbols are from Mayeda and Walter (1996), and indicate events from the Western US for which the coda-based spectra contained at least the $70 \%$ of the total energy, extrapolated to DC and to infinity. Light green symbols refer to estimates of energy given by Abercrombie (1995) for small earthquakes recorded in a deep borehole at Cajon Pass. 
The Orowan stress drop is a measure of the dynamic stress drop acting on the fault

plane during rupture $\left(\Delta \sigma_{d}=\frac{2 \mu E_{S}}{M_{0}}\right)$. In Figure 4 we see a clear increase of this parameter as the moment increases. It was interesting to note that the radiated energies obtained from earthquakes recorded in different parts of the world follow a similar trend, indicating a fundamental feature of rupture mechanics that can be explained by a number of processes (e.g, elastodynamic lubrication, Brodsky and Kanamori, 2001; fluid pressurization, Mase and Smith, 1985, 1987; melt on the fault plane, Kanamori and Heaton, 2000, etc.).

\section{Site Responses and Spectral Ratios}

Given the attenuation function provided by Malagnini et al. (2002) for this region, and the absolute moment-rate spectra derived in this study, we can estimate the "absolute" site terms for all the FVGSN stations (a site term for each component of the ground motions). In order to do so, we transformed the 206 absolute moment-rate spectra to ground velocity Fourier spectra, and we "propagated" them $40 \mathrm{~km}$ away from the hypocenters through equations (3), (4), and (5a,b) by Malagnini et al. (2002). Spectra were also resampled at their set of central frequencies. The arbitrary propagation of the moment-rate spectra to the reference hypocentral distance of $40 \mathrm{~km}$ has no effect on the results discussed in this paper. As explained in the study by Malagnini et al. (2002), the constraint of null attenuation term at an arbitrary reference distance $\left(D\left(r=r_{r e f}, f\right)=0\right)$ was introduced in order to make their system of linear equations nonsingular. Since we use their attenuation function and the same matrix inversion scheme, the "propagation" of our spectra to the mentioned reference distance is needed.

By using the formula:

$$
A M P_{k}\left(f, r_{i j}\right)=\operatorname{SOURCE} E_{j}\left(f, r=r_{r e f}\right)+\operatorname{SITE} E_{i}(f)+D\left(r_{i j}, r_{r e f}, f\right)
$$

the logarithm of the observed Fourier amplitude, $A M P_{k}\left(f, r_{i j}\right)$, is written as the sum of the contributions of three quantities: i) the absolute source spectrum, ii) the regional attenuation, $D\left(r_{i j}, r_{r e f}, f\right)$, and iii) the absolute site term, $S I T E_{i}(f)$. The latter represent the only unknowns of the problem. As already done in previous works (e.g., Malagnini et al, 2000 
2002), a hybrid approach is chosen: while robust estimates of the parameters are obtained at each frequency by performing an $\mathrm{L}_{1}$-norm regression, the related uncertainties are computed by inverting the problem in the least squares sense.

Our definition of the expression "absolute site term", which we are using throughout this section, relies on our ability to waveform model a few "good" earthquakes in the data set. Waveform modeling allows us to obtain a set of frequency-dependent corrections to our nondimensional "coda" spectra. Corrections would be with respect to site conditions and to Sto-coda transfer functions: large earthquakes provide low-frequency corrections, below their corner frequencies, whereas smaller events are responsible for the intermediate-frequency ones, still below their corners. Finally, empirical Green's functions link intermediate- and high-frequencies, provided that the EGF's are truly characterized by flat spectra up to frequencies outside the available band. Since corrections are tied to one another, from lowto high-frequency, uncertainties in the low-frequency amplitudes, relative to the waveform modeling, "propagate" through the entire bandwidth in form of an (unknown) dc-shift of the "absolute" site response at all frequencies. This unknown term is related to the uncertainties associated to the moments of the "reference" earthquakes. In terms of moment magnitudes, such uncertainty is typically about $\pm 0.2-0.3$ : in actual amplitudes of the ground motion, a factor of two is likely to represent a good guess.

Figure 5 shows the horizontal site terms (vector sum of radial and transverse terms) for the four stations with three-component sensors (BAD, CAE, DRE, and ZOU) the vertical terms, the horizontal-to-vertical ratios (four frames in the middle), and the classic spectral ratios between the vector sum of the horizontal site terms, with respect to BAD. The horizontal-to-vertical ratios for station ZOU is almost perfectly flat (Figure 6 and also Figure 10 by Malagnini et al., 2002). Based on this observation, most seismologists would have considered ZOU as a good reference location, under the hypothesis that a flat H/V ratio clearly means "no site effect". On the contrary, Figure 6 indicates that ZOU is strongly affected by a deamplification at high frequency, and that this phenomenon affects the vertical and the horizontal motion in the same way. The $\mathrm{H} / \mathrm{V}$ ratios computed for the other stations suggest 
absolute behaviors very different from those shown in Figure 6.

For each station, the H/V ratio shown in Figure 6 was computed on the 206 events only, whereas the corresponding two (one for each horizontal component) shown in Figure 10 of Malagnini et al (2002) refers to the results obtained over the entire data set of over 1800 events. Although, for a given station, the details of the single $\mathrm{H} / \mathrm{V}$ ratio obtained in this paper may be a little different from the two estimates computed by Malagnini et al. (2002) (one for each horizontal component of the ground motion), our results, in terms of amplitudes and shapes of the functional forms at all frequencies, are absolutely equivalent to theirs. The factor of $\sim \sqrt{2}$ between our single estimates and each of the two ratios per station shown in Figure 10 of Malagnini et al. (2002) is due to the fact that our H/V spectral ratio is taken between the vector sum of the two horizontal components, and the corresponding vertical component, of the absolute site terms. Malagnini et al. (2002), on the contrary, showed the single-component $\mathrm{H} / \mathrm{V}$ ratios (radial/vertical and transverse/vertical) of the inverted site terms for each station.

The parameter $\kappa_{0}=0.045 \mathrm{sec}$ found by Malagnini et al. (2002) controls the the highfrequency decay of the average vertical site effect of Figure 5. This value is relatively large, since it is defined to 'well-behaved' rock sites (Malagnini et al., 2002). We expect this characteristics to be responsible for the high-frequency deamplification that is observed at rock sites (Figure 5).

Spectral ratios between different site responses are easier to understand than absolute site effects. From the visual inspection of the absolute site terms of Figure 5 we chose the station BAD as our reference. We took the ratios between the vector sum of the horizontalcomponent terms of each station and the analogous vector sum computed at BAD. On average, all ratios are well-behaved between $0.5 \mathrm{~Hz}$ and $1.5 \mathrm{~Hz}$ (Figure 5), since they all tend to a unit value at the lowest frequency available. The steep rise at high-frequency for the ratio DRE/BAD is due to the anomalous behavior of BAD, at frequencies higher than 6.0 Hz. A similar phenomenon (of slightly higher intensity) was observed when we tried the other limestone site CAE as the reference station. We conclude that spectral ratios using 
"hard rock sites" only indicate relative site effects, and do not necessarily provide estimates of absolute site behaviors.

The absolute vertical site term for a station located on gravels (UDI) is very interesting (Figure 7), with a light amplification level at low frequencies $(f \leq 1.0 \mathrm{~Hz})$ due to the combination of the impedance gradients/contrasts that may exist at the site, and of the strong high-frequency attenuation (high $\kappa$ ) that takes place where the seismic velocities are lowest. Unfortunately, there is no direct geotechnical information for the stations used in this paper. Note that all site terms are affected by relatively large uncertainties at the sampling frequency of $0.5 \mathrm{~Hz}$ (Figures 5 and 7 ), a direct consequence of the characteristics of the sensors used and the low signal-to-noise ratio at this frequency for small earthquakes.

\section{Conclusions}

The present study complements the one by Malagnini et al. (2002) on the characteristics of the ground motions in the eastern Alps: whereas that work proposed a functional form describing the high-frequency crustal propagation $(f \geq 1.0 \mathrm{~Hz})$ as a function of frequency and hypocentral distance, this paper focuses on source radiation and uses the information on excitation and propagation of seismic waves to quantify absolute site effects.

Unbiased moment-rate spectra for 206 events recorded by the FVGSN were obtained by using a technique developed by Mayeda and Walter (1996), and subsequently modified by Mayeda et al. (2003). Spectra were then transformed into ground velocity and "propagated" to the arbitrary reference distance of $40 \mathrm{~km}$ through the application of the functional form developed for the same region by Malagnini et al. (2002). The resulting "excitation terms" were cast into a matrix form with the observed Fourier amplitudes recorded at the corresponding hypocentral distances, the empirical regional propagation terms, and the unknown site responses. The martix was inverted for all the unknown site terms, which represent the "absolute" responses for the FVGSN stations.

Here are our conclusions:

- i) apparently good rock sites may substantially distort the recorded seismic spectra; 
- ii) flat $\mathrm{H} / \mathrm{V}$ ratios do not mean that the corresponding locations are free from distortions induced by the shallow geology. $\mathrm{H} / \mathrm{V}$ spectral ratios do not necessarily quantify/describe "absolute" site behavior;

- iii) classic spectral ratios to a reference rock site cannot be interpreted in terms of absolute site effects: high-frequency amplifications indicated by traditional spectral ratios may be biased by rock-site deamplifications;

In this study we obtained very accurate moment-rate spectra, which were used for the computation of radiated energies. When normalized to seismic moment, radiated energy is proportional to dynamic stress-drop, $\Delta \sigma_{d}$. In the eastern Alps, as moment magnitude increases, $\Delta \sigma_{d}$ follows the same increasing trend indicated by results obtained by Abercrombie (1995) on small earthquakes recorded in a deep borehole at Cajon Pass, and by Mayeda and Walter (1996) on events of the Western US.

\section{References}

Abercrombie, R. (1995). Earthquake source scaling relationships from -1 to $5 M_{L}$ using seismograms recorded at 2.5-km depth, J. Geophys. Res., 100, 24015-24036.

Akinci, A., L. Malagnini, R.B. Herrmann, N.A. Pino, L. Scognamiglio and H. Eyidogan (2001). Predictive relationships for the ground motion in the Erzincan region (Turkey), Bull. Seism. Soc. Am., 91, 1446-1455.

Anderson, J.G., Y. Lee, Y. Zeng and S. Day (1996). Control of strong motion by the upper 30 meters, Bull. Seism. Soc. Am., 86, 1749-1759.

Anderson, J.G., and S. Hough (1984). A model for the shape of the Fourier amplitude spectrum of acceleration at high frequencies, Bull. Seism. Soc. Am., 74, 1969-1994.

Boore, D.M., W.B. Joyner, and T.E. Fumal (1993). Estimation of response spectra and peak acceleration from western United States earthquakes: an interim report, U.S. Geol. Surv. Open-File Rep. 93-509, 72 pp.

Boore, D.M., W.B. Joyner, and T.E. Fumal (1994). Estimation of response spectra and peak acceleration from western United States earthquakes: an interim report, Part 2, U.S. Geol. 
Surv. Open-File Rep. 94-127, 40 pp.

Borcherdt, R.D. (1994). Estimates of site-dependent response spectra for design (methodology and justification), Earthquake Spectra, 10, 617-653.

Borcherdt, R.D. (1992). Simplified site classes and empirical amplification factors for sitedependent code provisions, in Proc. NCEER, SEAOC,BSSC Workshop on Site Response during Earthquakes and Seismic Code Provisions, November 18-20, University of Southern California, Los Angeles, California.

Brodsky, E.E. and H. Kanamori (2001). Elastodynamic lubrication of faults, J. Geophys. Res., 106, 16357-16374.

Herrmann, R.B. (2002). Computer Programs in Seismology, Version 3.20, released on September 2, 2002, http://www.eas.slu.edu/People/RBHerrmann/CPS32.html.

Ide, S. and G.C. Beroza (2001). Does apparent stress vary with earthquake size?, Geophys. Res. Lett., 28, 3349-3352.

Kanamori, H. and T.H. Heaton (2000). Microscopic and macroscopic physics of earthquakes, GeoComplexity and the Physics of Earthquakes, Geophysical Monograph 120, AGU, 284 pp. King, J.L., and B.E. Tucker (1984). Observed variations of earthquake motion across a sediment-filled valley, Bull. Seism. Soc. Am., 74, 137-151

Malagnini, L., R.B. Herrmann, M. Di Bona (2000). Ground-Motion Scaling in the Apennines (Italy), Bull. Seism. Soc. Am., 90, 1062-1081.

Malagnini, L., A. Akinci, R.B. Herrmann, N.A. Pino and L. Scognamiglio (2002). Characteristics of the ground motion in Friuli (Northeastern Italy), Bull. Seism. Soc. Am., 2186-2204.

Mayeda, K., A. Hofstetter, J.L. O’Boyle, and W.R. Walter (2003). Stable and transportable regional magnitudes based on coda-derived moment-rate spectra, Bull. Seism. Soc. Am., 93, 224-239.

Mayeda, K. and W.R. Walter (1996). Moment, energy, stress drop, and source spectra of western United States earthquakes from regional coda envelopes, J. Geophys. Res., 101, 11195-11208. 
Martin, G.R. and R. Dobry (1994). Earthquake site response and seismic code provisions, NCEER Bull., 8, 1-6.

Mase, C.W. and L. Smith (1985). Pore-fluid pressures and frictional heating on a fault surface, Pure and Applied Geophysics, 122, 583-607.

Mase, C.W. and L. Smith (1987). Effects of frictional heating on the thermal, hydrologic, and mechanical response of a fault, J. Geophys. Res., 92, 6249-6272.

Papageorgiou, A.S. and K. Aki (1983). A specific barrier model for the quantitative description of inhomogeneous faulting and the prediction of strong ground motion. Part II. Applications of the model, Bull. Seism. Soc. Am., 73, 953-978.

Randall, G. R., C. J. Ammon and T. J. Owens (1995). Moment-tensor estimation using regional seismograms from a Tibetan Plateau portable network deployment, Geophys. Res. Letters, 22, 1665-1668.

Raoof, M., R.B. Herrmann and L. Malagnini (1999). Attenuation and excitation of threecomponent ground motion in Southern California, Bull. Seism. Soc. Am., 89, 888-902.

Richards, P.G. (1976). Dynamic motions near an earthquake fault: a three-dimensional solution, Bull. Seism. Soc. Am., 66, 1-32.

Shang and Gao (1988). Transportation theory of multiple scattering and its application to seismic coda waves, Sci. Sinica, Series B, 31, 1503-1514.

Steidl, J.H., A.G. Tumarkin, and R.J. Archuleta (1996). What is a reference site?, Bull. Seism. Soc. Am., 86, 1733-1748.

Tucker, B.E. and J.L. King (1984). Dependence of sediment-filled valley response on input amplitude and valley properties, Bull. Seism. Soc. Am., 74, 153 - 165 


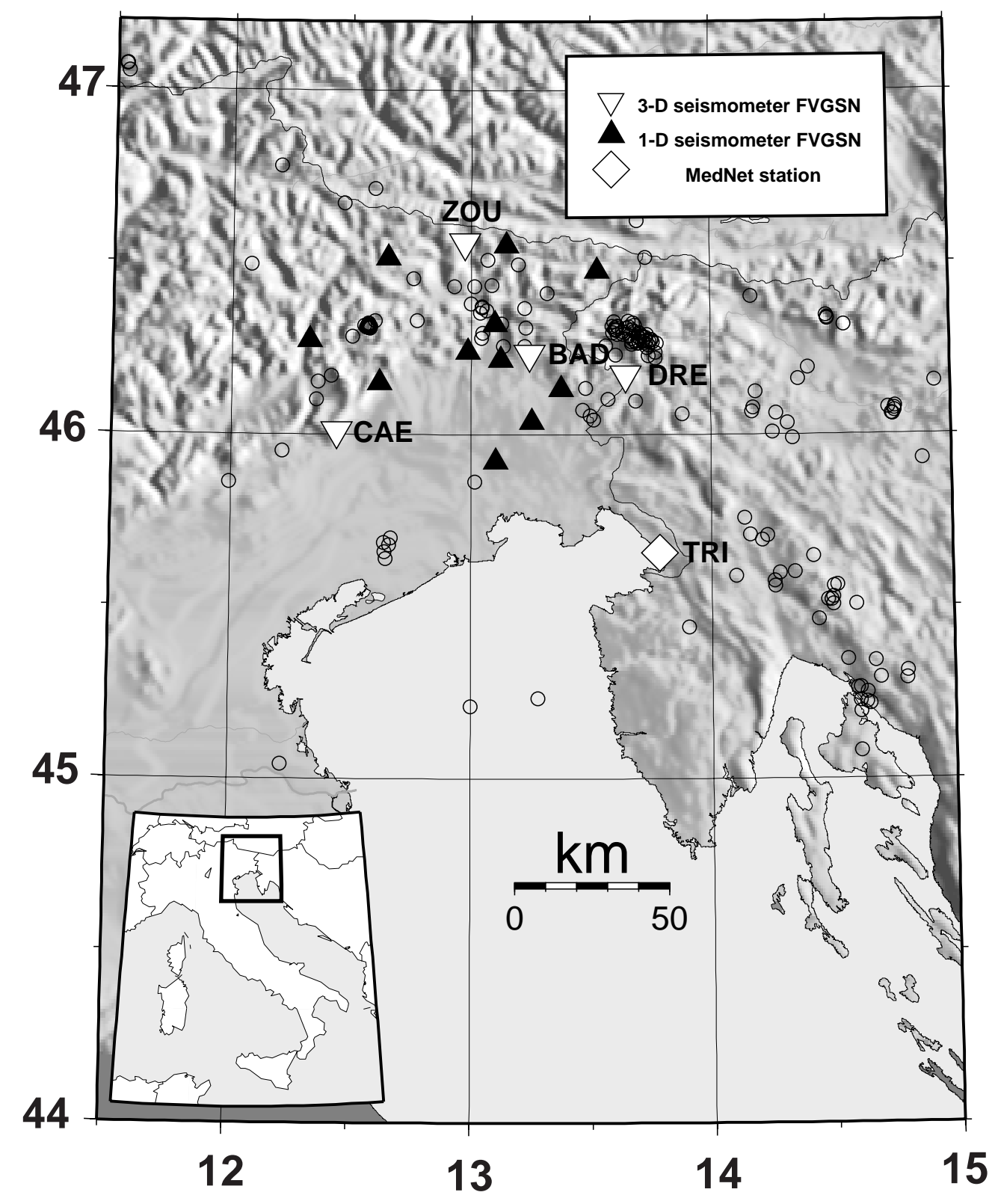

Figure 1: The map shows the stations and the 206 events used in this study. Three-component stations are indicated by reversed triangles, whereas black triangles are used for single-component (vertical) seismometers. The diamond locates the MedNet station TRI. Open circles show the epicenters of the seismic events. Names of stations are indicated only for the three-component ones. After 1998, the configuration of the FVGSN has changed, with a number of single-component instruments upgraded to three-component, and a few new sites. 

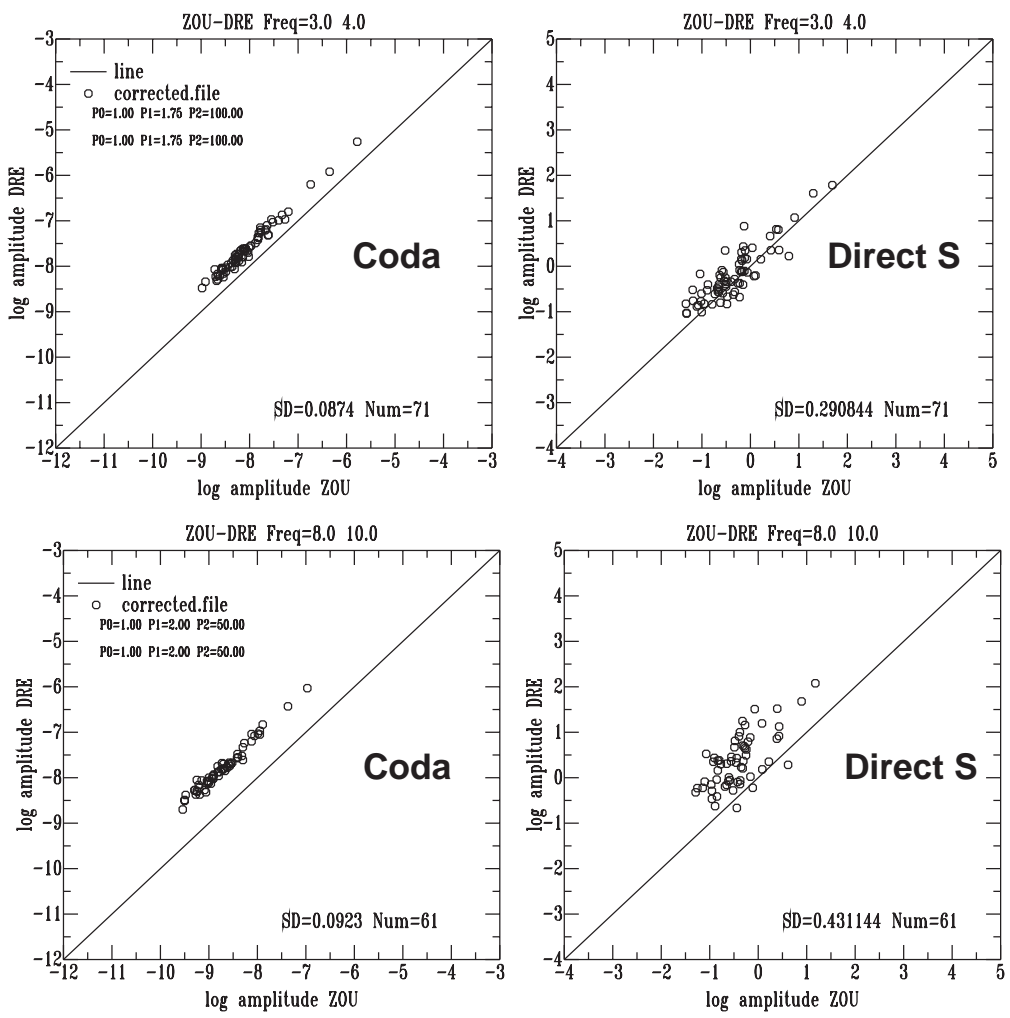

Figure 2: Interstation plots (station pair ZOU-DRE) for coda-derived amplitudes (first column) and for direct-wave amplitudes (second column), corrected for hypocentral distance and anelastic attenuation. The upper two frames refer to the 3.0-4.0 Hz sampling frequency band, whereas the lower two frames refer to the $8.0-10.0 \mathrm{~Hz}$ one. Indicated in each frame are the number of data points and the standard deviation with respect to the average interstation distance from the best line parallel to $\mathrm{y}=\mathrm{x}$. Note the substantial decrease of the interstation standard deviations when coda amplitude measurements are used. For each comparison between direct-wave and coda interstation plots, the set of data points is the same. 


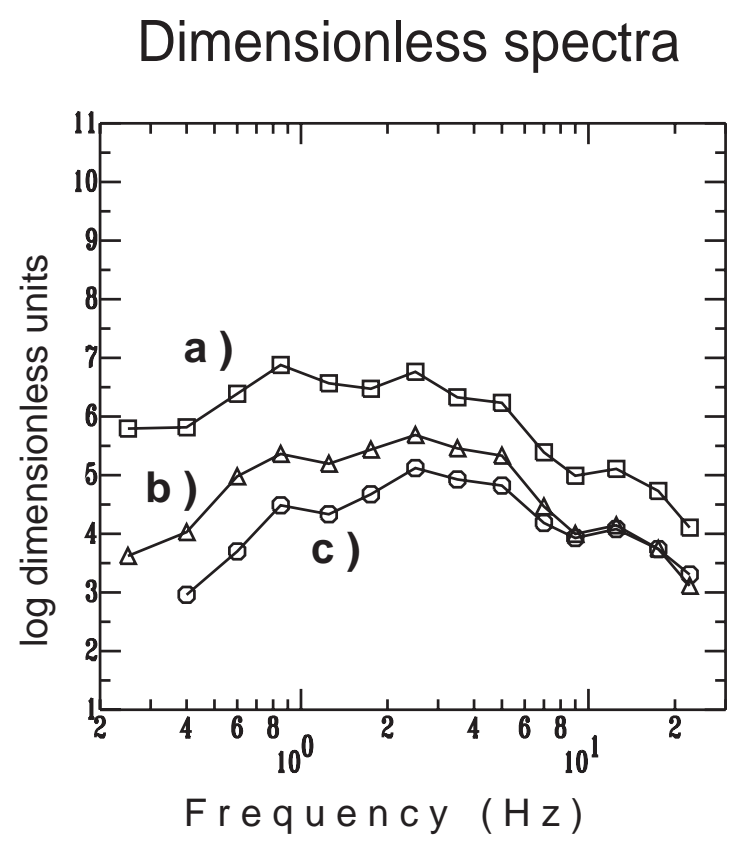

Moment-rate spectra

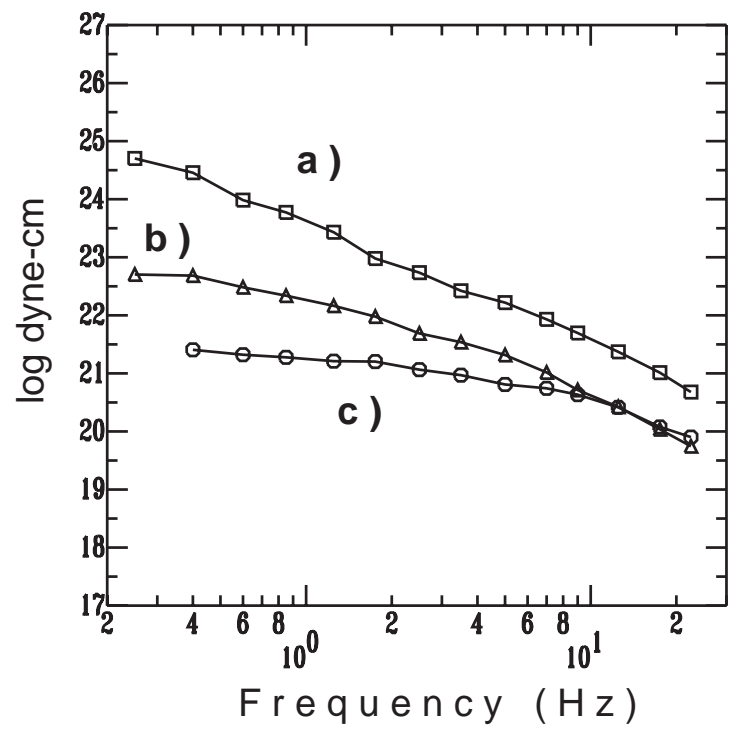

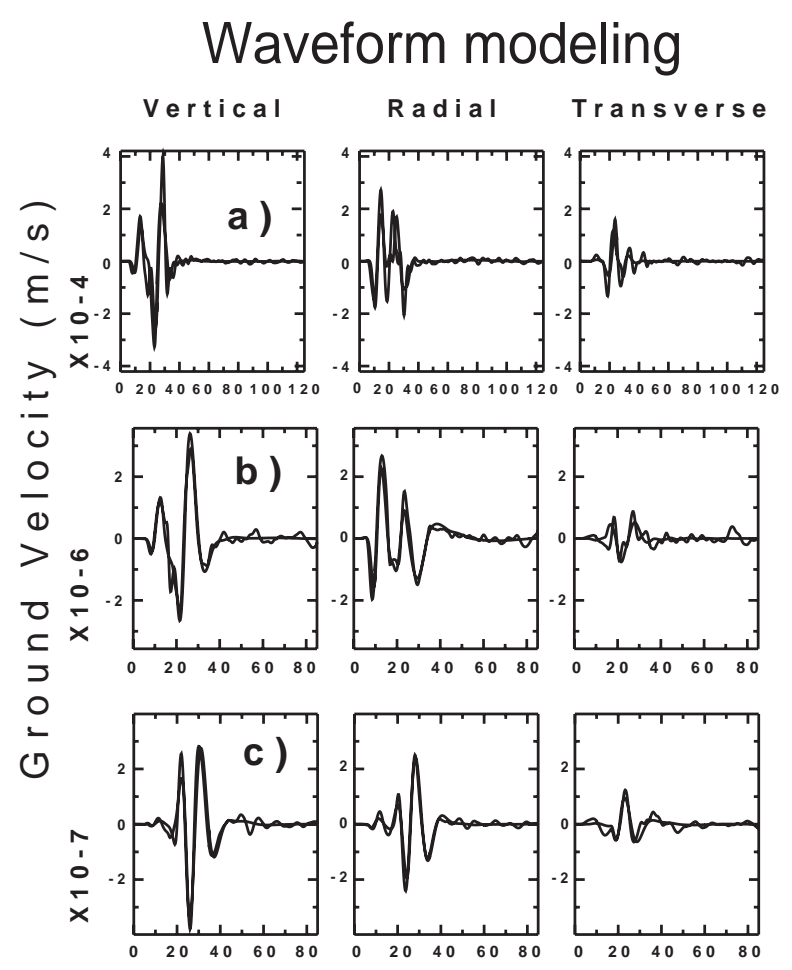

Time ( s e c )

Figure 3: Upper left frame: dimensionless spectra at station BAD for three events of the data set for which broadband recordings from the MedNet station TRI were available (see Figure 1). (a) April 12 (102) 1998 10:43:16, Mw 5.59 Depth=5.0 Km, Strike=313, Dip=80, Rake=-165, Fmin=0.01, and Hz Fmax=0.1 Hz; (b) May 6 (126) 1998 02:39:51, Mw 4.29, Depth=3.0 Km, Strike=53, Dip=60, Rake=50, Fmin=0.02 Hz, and Fmax=0.1 Hz; (c) May 28 (148) 1998 09:32:03, Mw 3.45 Depth=9.0 Km, Strike=50 Dip=81 Rake=74, Fmin=0.01 Hz, and Fmax=0.1 Hz. Upper right frame: waveform modeling of the events. Lower frame: moment-rate spectra of the three events. 

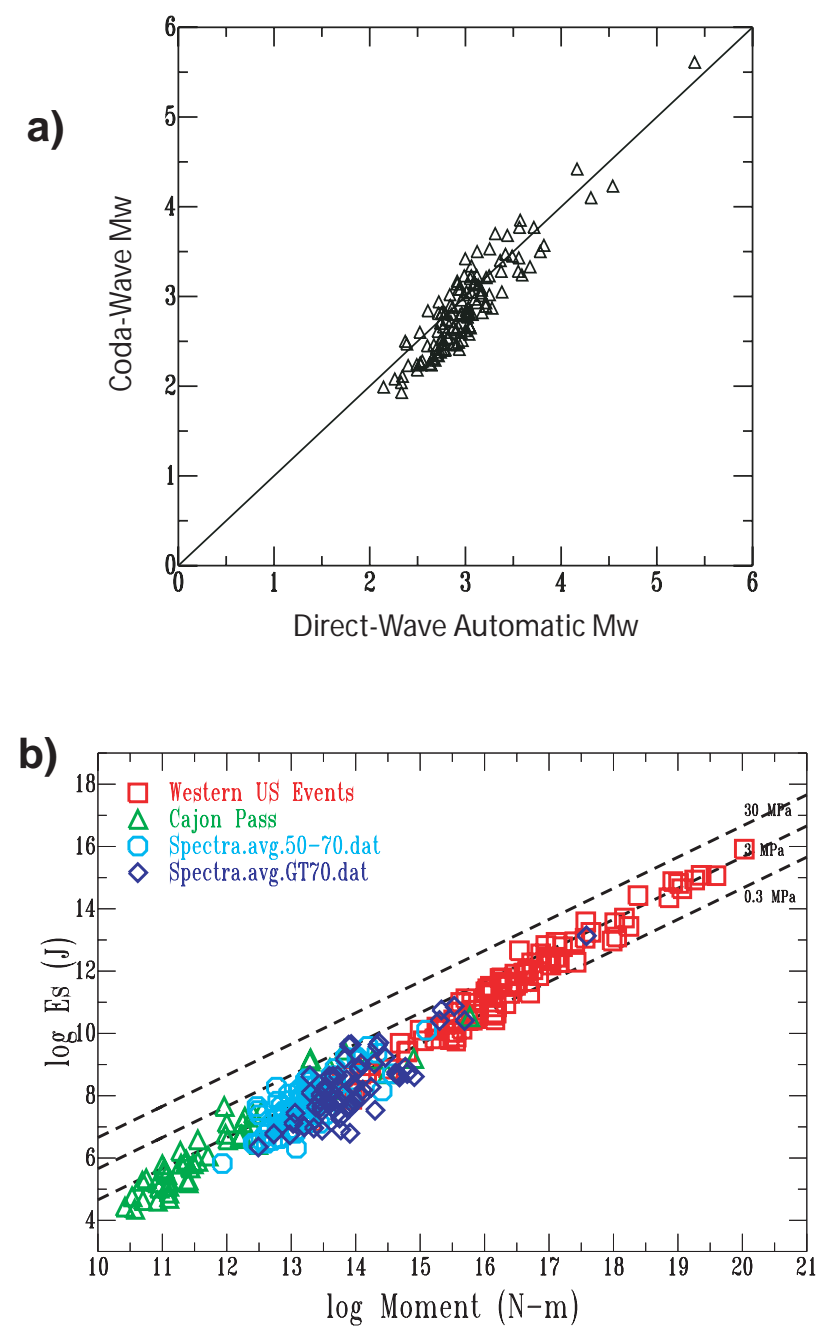

Figure 4: a: Automatic moment magnitudes (y-axis) computed for all the 206 events in the data set by using the crustal attenuation computed by Malagnini et al. (2002) for Northeastern Italy, vs. the corresponding coda-based moment magnitudes (x-axis). b: radiated seismic energy $E_{S}$ is plotted vs. seismic moment. Energies recovered over the $70 \%$ of their total values for the eastern Alps are plotted in deep blue. Light blue data points correspond to earthquakes for which momentrate spectra did show a corner frequency (recovered radiated energies between $50 \%$ and $70 \%$ of the total). Other data points were computed by Mayeda and Walter (1996) (red) and by Abercrombie (1995) (green). Lines of constant dynamic stress drop (0.3, 3, $30 \mathrm{MPa})$ are also shown. Data from the eastern Alps seem to follow the same trend indicated by their combined results obtained for the western US (red) and Cajon Pass (green). 

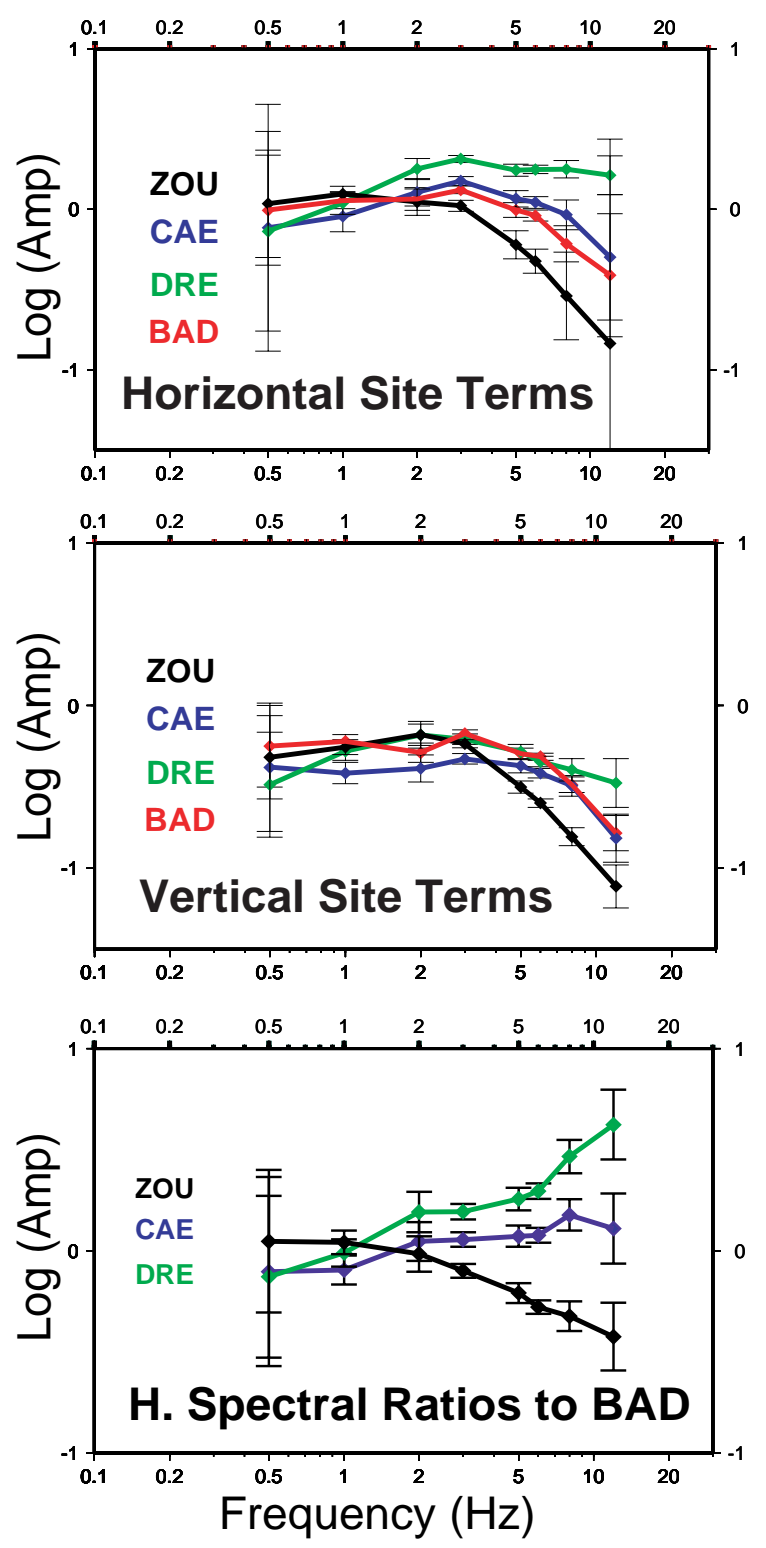

Figure 5: Up) absolute horizontal site terms for stations DRE (marly sandstone, etc.), CAE (limestone), BAD (limestone), and ZOU (dolomite); middle) absolute vertical site terms for DRE, CAE, BAD, ZOU; bottom) horizontal spectral ratios to reference site BAD (ratios between the quantities shown in the uppermost frame). 


\section{Horizontal/Vertical Ratios}

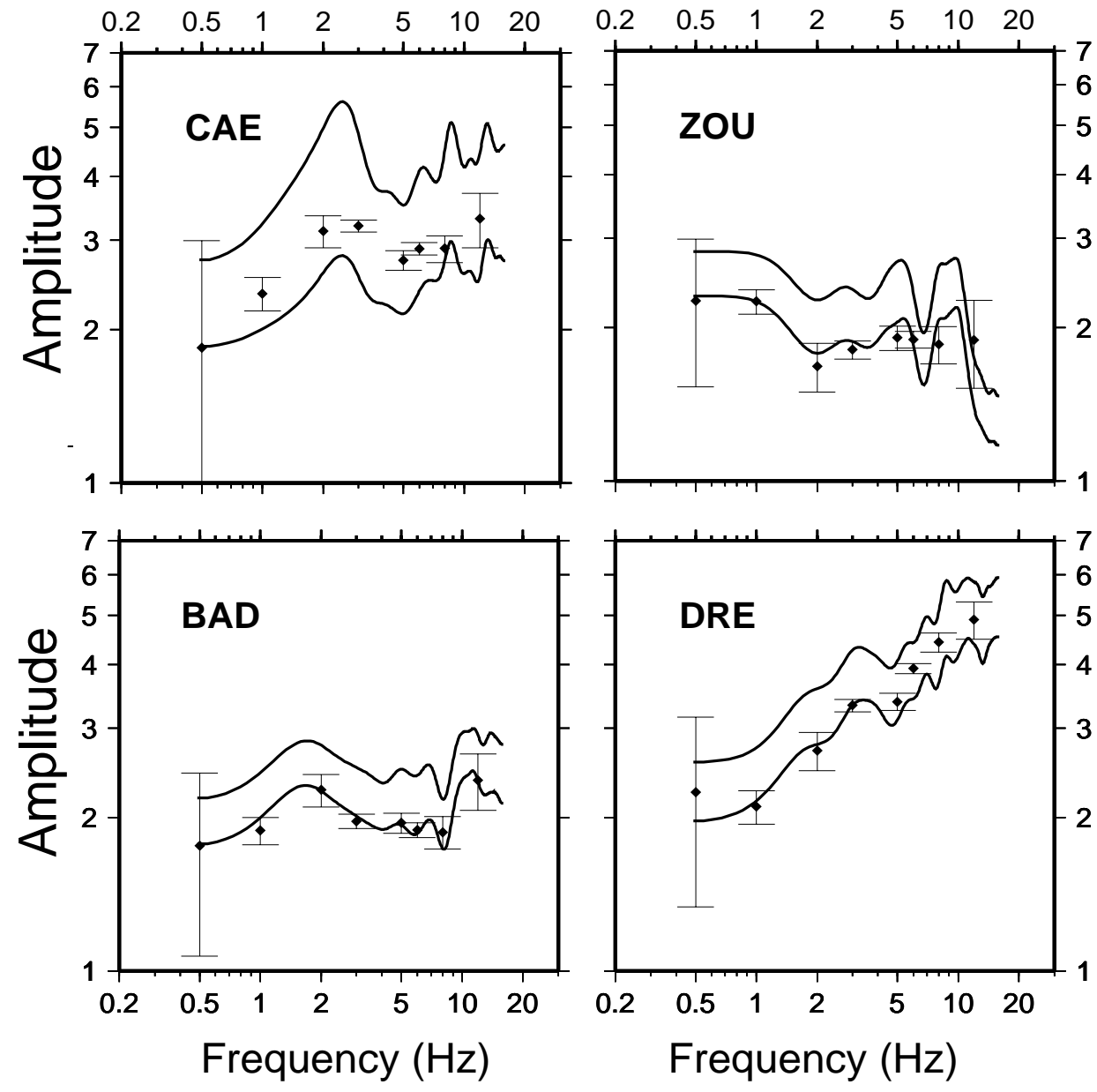

Figure 6: Horizontal-to-vertical ratios at stations BAD, CAE, DRE, ZOU, computed directly from the recorded waveforms of the 206 events of this data set, and from the quantities shown in the left set of frames of this figure. Fourier spectra were computed using 14 sec-long windows, starting 2 sec before the S-wave picks, and the vector sum of the horizontal components was computed. Before taking the ratios, horizontal and vertical spectra were log-smoothed. Average spectral ratios plus and minus one standard deviation are plotted with solid lines. Error bars on spectral ratios are obtained from those computed during the regressions. 


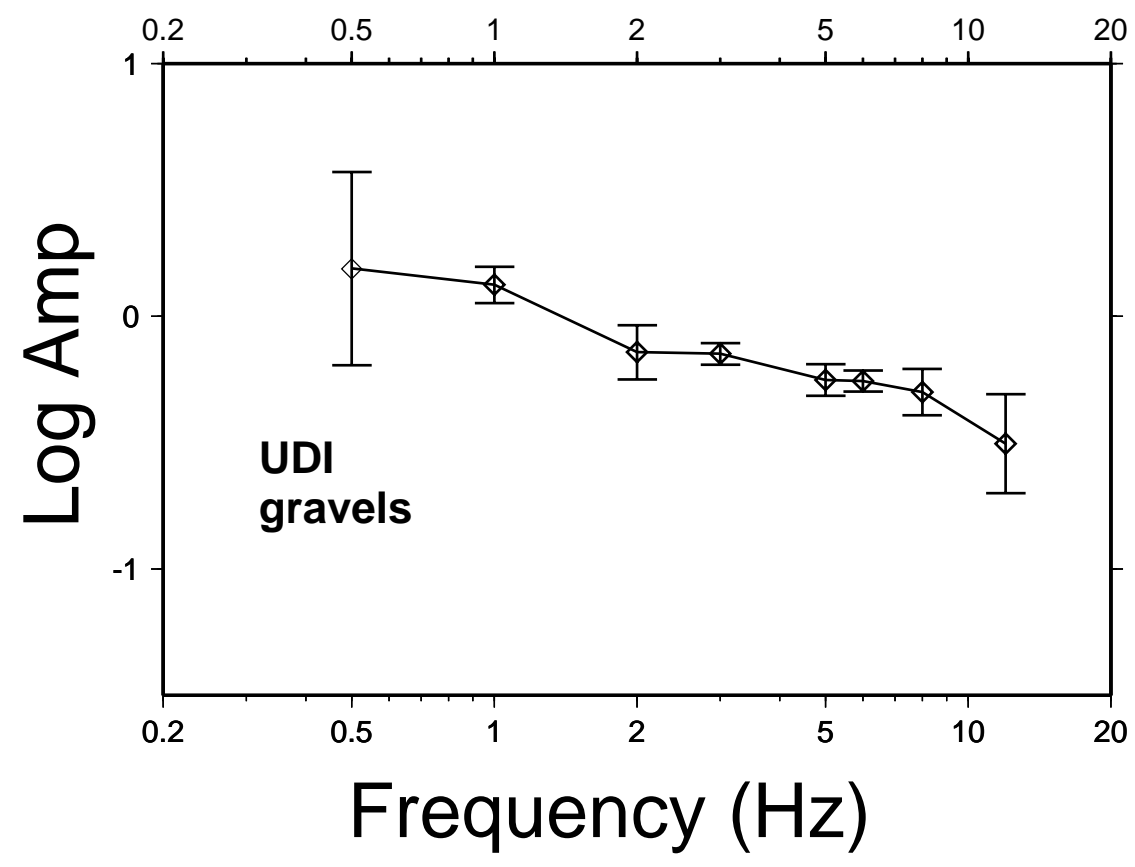

Figure 7: Absolute vertical site term for station UDI (gravel). 
University of California

Lawrence Livermore National Laboratory

Technical Information Department

Livermore, CA 94551

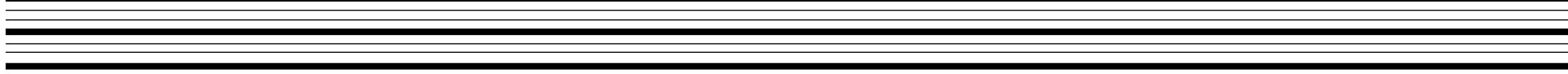

